\title{
Support for Urgent Computing Based on Resource Virtualization ${ }^{\star}$
}

\author{
Andrés Cencerrado, Miquel Ángel Senar, and Ana Cortés \\ Departamento de Arquitectura de Computadores y Sistemas Operativos \\ 08193 Bellaterra (Barcelona), Spain \\ andres.cencerrado@caos.uab.es \\ \{miquelangel. senar, ana.cortes\}@uab.es
}

\begin{abstract}
Virtualization technologies provide flexible execution environments that could bring important benefits for computational problems with strong deadlines. Large Grid infrastructures are becoming available nowadays and they could be a suitable environment to run such on-demand computations that might be used in decision-making processes. For these computation, we encounter the need to deliver as much resources as possible at particular times. These resources may be provided by different institutions belonging to a grid infrastructure but there are two important issues that must be satisfied. Firstly, all resources must be correctly configured and all the components needed by the application must be properly installed. If there is something small missing that is required then applications will fail. Secondly, the execution of urgent applications must be made quickly in order to produce useful results in time. If applications must wait in a queue, results might be useless because they are obtained too late. To address these issues, we describe a job management service, based on virtualization techniques, that avoids configuration problems and increases the number of available resources to run applications with critical deadlines. We describe the main components of our service that can be used on top of common batch queue systems and we show some experimental results that prove the benefits of applying time-sharing techniques on the virtual machines to increase the performance of urgent computations.
\end{abstract}

\section{Introduction}

Nowadays, scientific community rely on computational resources in order to solve most of the present scientific problems. In order to satisfy computing-intensive problems in the minimum possible time, since the beginning of the $90 \mathrm{~s}$ it is possible to resort to distributed computing, because of the deployment of grid infrastructures. These kind of infrastructures allow the users to count on many computing resources managed in a decentralized way.

Besides, there is an emerging area in the scientific applications field, Urgent High Performance Computing (Urgent HPC), which requires a great capacity

\footnotetext{
* This work is supported by the MEC-Spain under contracts TIN 2007-64974.
}

G. Allen et al. (Eds.): ICCS 2009, Part I, LNCS 5544, pp. 227-236, 2009.

(C) Springer-Verlag Berlin Heidelberg 2009 
of computing resources at a given time. Urgent HPC applications could take benefit from a grid environment prepared to respond efficiently, because of the great resources offer and variety it can supply. In fact, there are several projects in progress which main goal is to get response from a multiple-resource sites as quick as possible [1], but due to the grid environments nature, it is necessary to propose new management strategies that provide not only a quick response, but a more efficient use of the available resources for the results to be more accurate in a shorter period of time.

Grid computing can be considered as a consolidated field in high performance computing, however, it still presents serious limitations from the point of view of Urgent HPC requests. Response time is an usual handicap in such environments. Each administrative domain, in a grid infrastructure, has its own components that take care about security and scheduling issues. All these components introduce considerable delay to the jobs starting, which is a clear penalty for urgent computing applications.

Nevertheless, time response is not the only constraint, compatibility between the application to be launched and the execution platform is a very important handicap as well. Libraries, permissions and the host operating system are examples of factors that can compromise the compatibility and, in consequence, the successful execution of the jobs. Many times, users are not aware of the requirements of their own jobs. This kind of ignorance usually leads to a considerable waste of time trying to guarantee the correct execution of the application on the remote sites and sometimes it turns out to be an impossible task.

Moreover, most of scientific applications rely on models/simulators that do not provide accurate results and the quality of the results will depend on how many times the underlying simulation can be executed before a deadline. Therefore, in those cases, as much executions we can deliver at a given time, the better the results become. Consequently, we focus on increasing the number of suitable working nodes for the underlying urgent application at a given time independently on the sites' particular configuration.

Virtual Machines (VMs) are becoming popular in grid computing as they provide a way to abstract the grid resources and allow grid applications to be run without worrying about the underlying platform of the resource. In this work, we propose a job-management service based on virtualization techniques, that avoid configuration problems and increases the number of available resources to run applications with critical deadlines.

This paper is organized as follows. In the next section, the main features of virtual machines are described. In section 3, we describe the architecture of the job management architecture. The experimental study is reported in section 4 and, finally, the main conclusions are included in section 5 .

\section{Virtual Machines for Urgent Computing}

As we have previously mentioned, virtual machines provide an abstraction of the underlying computing resources allowing any kind of application to be executed 
without misleading problems. In particular, VMs provide several important advantages:

a) Physical platform isolation: thanks to the abstraction carried out by the virtual machine monitor (VMM), tasks execution does not depend on the host device features. Thus, any VM instance on which user can execute his application successfully will be useful and, therefore, any working node (WN) in the site can satisfy application demands.

b) Multiplexation capability: depending on the features of the host platform, it can support multiple VM instances execution, which could provide better performance per WN.

c) Possibility of deploying new subpool of WNs: a VM, like a real physical machine, can execute the software needed to become a new WN into the site, able to receive and process different tasks as well as physical WNs do. Furthermore, the fact of having multiple VMs as a part of the working nodes set of the site, allow us to deploy a new subset, or subpool, of virtual WNs. The idea besides that is the one referred as to VM recycling. Instead of starting and stopping a VM each time the submitted job requires this kind of execution environment, VM recycling deals with the idea of not stopping the VM and keeping it alive waiting for new tasks to be executed. This recycling strategy saves time, as the time needed to instantiate the VM is skipped.

As one can see, these features solve, or at least relieve, the main constraints of grid environments when dealing with an urgent HPC application. How each one of the above mentioned handicaps are overcome is subsequently listed.

- Compatibility constraints elimination: in order to be able to execute the application in any grid site, it is sufficient to have a VM that emulates a trusted system on which the desired application executes successfully.

- Response time reduction: as it has been previously mentioned, VM recycling permit to deploy subpools of virtual WNs that could be managed in a different way than the physical ones, establishing, for example, different priority polices, even up to dedicating it exclusively for particular applications.

- Turnaround time reduction: because of the multiplexation capability, it is possible to carry out more executions per time unit, reducing the turnaround time of each individual task/application execution compared to a standard batch execution.

As stated previously, this work is focused on efficient managing strategies of the grid-available resources. To achieve this goal, we propose a grid site local-level architecture design based on the use of Virtual Machines (VMs), as a suitable platform for grid infrastructures in order to allow an efficient and successful processing of urgent scientific applications under such environments.

Although this approach seems very promising, it also raises new difficulties and new challenges that must be explored, for instance, it is needed an accurate analysis of possible performance degradations as well as collateral effects. These aspects will be studied in the subsequent sections. 


\section{Architecture Design}

Virtual machines in grid computing can be implemented in different ways depending on the purpose and intention of the grid. We shall now briefly describe two of the most significant approaches according to our work:

1.-Virtual Machines as Grid Infrastructure: this method allows Grids to be set up and deployed easily. Grid middleware is installed and configured within virtual machines, and users can create and use a Grid infrastructure by installing and deploying these virtual machine images. Machines are added and removed from the Grid when users start and terminate their virtual machines. One example of this virtual machine grid computing implementation is Grid Appliance [2].

2.- Virtual Machines running on Grid Infrastructures: this method is based on the use of an already existing infrastructure for supporting grid computing. Virtual Machines are executed as processes using the grid middleware just like normal applications. Some examples are In-VIGO [3] and Maestro-VC 4].

Our proposal fits the second approach, taking into account the possibility of dealing with virtual resources as new WNs inside the site, as well. Figure 1] depicts a modular schema of the proposed architecture for an urgent job execution site based on virtual resources. In this approach, Grid middleware layer is based on EGEE gLite framework [5. As one can see, there are new resource components as VMs which have been deployed as a consequence of the execution of an ordinary task in a physical node, consisting of VM instance starting. These virtual nodes have the capability to become new WNs into the site. Our contribution, by means of this design, consists of the Virtual Spaces Job Manager (VSJM) component description, which is the responsible for the appropriate deployment and management of these virtual resources.

VSJM manages the virtual resources subpool, establishing direct connection with it, in order to be able to diversify efficiently incoming virtual-resource demanding jobs. This component is constituted by three subcomponents:

- Execution manager: responsible for carrying out scheduling tasks for this sort of jobs.

- VM manager: the subcomponent in charge of VM instantiation, by submitting a VM starting up job to the Local Resource Management System (LRMS), stopping, recycling and even migrating it.

- Job manager: this component will deal with the submission and control of tasks to be executed in virtual resources.

In order to include to the system the capability to correctly attend incoming requests, we lean on an extension of the EU-Datagrid Job Description Language [6], that is, the users, by means of a JDL file, may specify which virtual environment should be provided for their jobs. Figure 2 shows a simplified example of an extended JDL file where we include two new attributes: the VMId, which univocally specifies what VM image should be deployed, and the UrgencyLevel, 


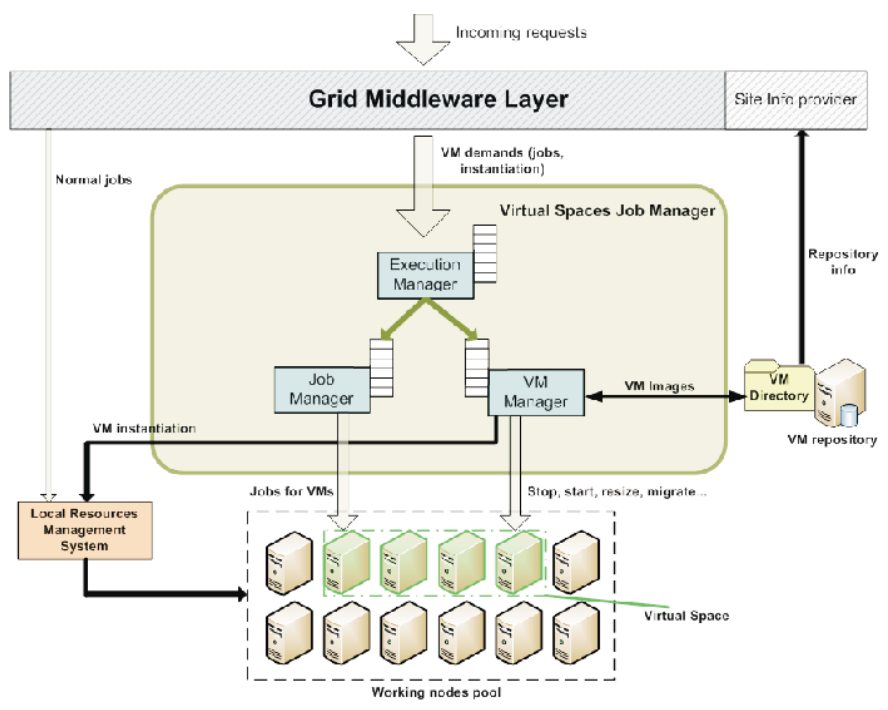

Fig. 1. Virtual resources-based site architectural design approach

Executable
Arguments
JobType
InputSandBox
VMId
UrgencyLevel

Executable

Arguments

JobType

InputSandBox

UrgencyLevel

$$
\begin{aligned}
& =\text { "fusion_app"; } \\
& ="-\mathrm{n} " ; \\
& =\text { "Normal"; } \\
& =\{\text { "fusion_app" }\} \\
& =\text { e97315e469fb; } \\
& =1 ;
\end{aligned}
$$

Fig. 2. Extended JDL job description

which specifies, in a 0 to 2 scale, the emergency degree of the incoming job, in order to efficiently attend this kind of jobs.

Based on this extended JDL file, users can order the instantiation of a VM image specifying its VMId, which had been established once the image had been deposited on the VM repository. Our objective is to adapt this component to the Globus Virtual Workspaces virtual repositories management system [7 8].

Thus, jobs get into the system through the Grid middleware layer, which determines whether jobs will be executed in a physical WN, steering them directly to the LRMS, or jobs will be executed in a virtual WN, steering them in this case to the VSJM module. Additionally, jobs that demand a virtual environment, which is not already deployed, will cause the VM manager to act submitting an ordinary job to the LRMS consisting of VM starting up.

This architectural approach constitutes a solution that provides useful services for urgent computing, from which outstand the following ones:

- Allowing usual job execution: new functions added by our system do not interfere in the way the site operated previously. 
- Allowing VM instances executions (offered by the site or supplied by the user): our approach contemplates the possibility of offering a series of VM images available in the site (stored in a storage element), and accepting user images as well, depending on authorization policies.

- VMs capability to become WNs and constituting a new WN subset: our system submits VMs images as normal jobs to the Local Resource Management System without requiring any special feature.

- Transparent job submitting to VM instances: our system includes non-intrusive techniques that allow the automatic instantiation of a resource management system (such as Condor [9]) when a VM image is started. This resource management system does not interfere with the Local Resource Management System deployed in the site and is used only by our Job Manager in order to submit jobs directly to VM instances. By exploiting disk images inclusion techniques, there is no need to modify the users' VM image nor to make them install any extra software in their images.

- Efficient VMs recycling management: the automatic deployment of a resource management system when each VM is started allows the execution of multiple jobs in the same VM instance. Therefore, significant saving of time is obtained by avoiding the overhead incurred in VM instantiation.

- Basic infrastructure that can be able to dynamically increase the number of resources in case of emergency: our system enables the development of intelligent scheduling strategies that can determine in an automatic and dynamic way when it is necessary to deploy new VM instances in order to attend to the incoming tasks load, without the need for the user to send VMs execution jobs.

\section{Performance Analysis and Experimentation}

This section describes some experimental results obtained with a first prototype that supports the basic functionality of the job management service described in the previous section. The goal is to demonstrate feasibility of the design and show the potential use of the virtual machine multiprogramming mechanism. Our prototype is interfaced to Condor as Local Resource Management System. The current stable version of Condor (7.0.4) provides direct support for managing VM images (which includes transferring images from a submission machine to an execution machine, starting and stopping the virtual machine, and so on). Condor was running on a testbed made of 12 Pentium IV machines, running Linux. The Grid middleware used for accessing the testbed was based on gLite. And we extended the existing gLite's job manager with additional services that provide a basic control of virtual images and application jobs.

We carried out a set of experiments with this prototype to measure the performance of two applications running in a virtualized mode and the effect of virtual machine multiprogramming on application performance. Our multiprogramming scheme allows the execution of two or more virtual machines on a physical machine. Each virtual machine can be used for a different application 
thus allowing urgent jobs to time share the CPU with other jobs running on a different virtual machine. A graceful degradation in performance is observed but this scheme does not require to kill or checkpoint a running job when an urgent job enters into the system.

We have used two computing-intensive applications to carry out the experimental analysis presented in this section. The first one is a Dynamic Data Driven Genetic Algorithm for fire spread prediction (DDDGA) 10. The second one is BLAST [11, a well known biology application, based on searching regions of local similarity between genetic sequences. Both applications are compute-intensive. However, in our experiments we have used DDDGA as an example of an urgent job that was time shared with a non-urgent job (BLAST).

There is no appreciable cost (in terms of execution time and overhead) derived from the use of VMs as computational tool instead of a physical machine in the case of compute-intensive applications [12. Table 1 shows execution times of both applications under VM and under native host.

Table 1. DDDGA and BLAST execution times (minutes)

\begin{tabular}{|l|l|l|l|}
\hline \multicolumn{2}{|c|}{ Native host } & \multicolumn{2}{c|}{ Virtual Machine } \\
\hline BLAST & DDDGA & BLAST & DDDGA \\
\hline 58.5 & 10 & 62 & 11 \\
\hline
\end{tabular}

We carried out another experiment to demonstrate the fact that it is possible to implement an adaptive scheduling system which is able to exploit virtual resources depending on the emergency or priority of the incoming tasks. As VMM we have used Xen [12], which provides useful techniques to control the VMs behavior, such as CPU limitation. The experiment presented in this work consists on evaluating how many executions of urgent incoming jobs (DDDGA) can be carried out in the same time of another task already in execution, without the need neither to suspend it nor to refuse the incoming requests, depending on the CPU limitation balance between the virtual working nodes instances. Results are shown on table 2. The time incurred by Condor to transfer the VM image is not included. table 2 only shows the actual time incurred in the execution of each job once the corresponding VM was started. It is worth noting that, according to our architecture design, each instance of a job does not require to run in a different VM. In general, only a small set of VM can be started and then multiple instances of the job will run there until the whole set of jobs is completed, thus limiting the overall overhead incurred in VM transfer and initialization. In this experiment, DDDGA and BLAST run simultaneously on the same machine but a different amount of CPU was given to each VM. The CPU amount ranged from a minimum of $10 \%$ to a maximum of $90 \%$ (for instance, first row in table 2 shows the results of DDDGA running in a VM that was limited to $10 \%$, while BLAST was running in a VM that was using $90 \%$ of the physical CPU). The same experiment was conducted on different machines of our testbed and table 2 shows average results. 
Table 2. CPU limitation experimental results (execution times in minutes)

\begin{tabular}{|l|l|l|l|l|l|l|}
\hline $\begin{array}{l}\text { CPU limit } \\
\text { distribution } \\
\text { DDDGA VM- } \\
\text { BLAST VM) }\end{array}$ & $\begin{array}{l}\text { \#DDDGA } \\
\text { executions }\end{array}$ & executions & $\begin{array}{l}\text { Whole } \\
\text { DDDGA } \\
\text { set execution } \\
\text { time }\end{array}$ & $\begin{array}{l}\text { Whole } \\
\text { BLAST } \\
\text { set execution } \\
\text { time }\end{array}$ & $\begin{array}{l}\text { DDDGA mean } \\
\text { execution } \\
\text { time }\end{array}$ & $\begin{array}{l}\text { BLAST mean } \\
\text { execution } \\
\text { time }\end{array}$ \\
\hline $10 \%-90 \%$ & 1 & 2 & 128 & 132 & 128 & 66 \\
\hline $25 \%-75 \%$ & 2 & 1 & 82 & 82.5 & 41 & 82.5 \\
\hline $50 \%-50 \%$ & 5 & 1 & 126 & 129 & 25.2 & 129 \\
\hline $75 \%-25 \%$ & 18 & 1 & 277 & 287 & 15.39 & 287 \\
\hline $90 \%-10 \%$ & 49 & 1 & 591 & 602 & 12.06 & 602 \\
\hline
\end{tabular}

As seen in table 2 , it is possible to get an important benefit by taking into account applications behavior in each case of CPU limitation. A hypothetic situation could be the fact of having a set of non-urgent BLAST jobs queued and/or in execution, and an urgent request for executing as many instances of DDDGA as possible. The results obtained indicate that if the system balanced properly the use of CPU on the virtual resources many urgent tasks could be completed while non-urgent ones will be kept in execution, without having to suspend them. For instance, when DDDGA was running with $90 \%$ of CPU, the overhead on the execution time of each job was nearly negligible, compared to the case where DDDGA was running alone (Table 1). A total of 49 instances of DDDGA were completed at the time taken by BLAST to complete one instance. Obviously, performance degradation of BLAST was significantly higher, but this is an acceptable situation in a scenario in which an urgent application arrives into the system and needs to harness as many computational resources as possible.

Further exploitation of this multiprogramming mechanism requires the study of new scheduling policies that make an efficient use of it in order to get the maximum performance for each node in the grid site. Open issues that can be explored in the future include:

a) To establish useful scheduling policies for virtual resources exploitation.

b) To satisfy, by means of these techniques, multiple time constraints (i.e. jobs deadlines) thanks to intelligent strategies based on real-time CPU and memory limitation for jobs in execution in order to provide a suitable environment for incoming tasks, respecting both the old jobs and the new jobs deadlines.

Furthermore, the addition of some sampling mechanism will enable our system to give feedback to the users about execution time estimations, taking into account how many resources could be devoted to their jobs in a given period of time, and how would be possible to resize them in terms of CPU limitation.

\section{Conclusions and Future Work}

This work addresses some of the problems related to execution of urgent jobs in a distributed computing environment. We have studied and proposed an 
architecture for a job management service, based on virtual resources, that local sites should present in order to attend successfully and efficiently incoming computing-intensive scientific applications. By using virtualization techniques, our system has several advantages because urgent codes will be able to run without worrying about applications' libraries and architecture dependencies. The system includes separated services that are responsible, on the one hand, for managing virtual machine instantiation and, on the other hand, for job execution on the corresponding virtual machine instances. One of our goals was to keep interoperability with already existing Local Resource Management Systems (LRMS) and cluster middleware. No changes are required to existing LRMS, and the only additional software required on each cluster are the necessary components needed by the underlying virtualization engine (Xen, VMware...).

A first prototype of our service has been built and tested on a cluster managed by Condor. Several experiments were conducted to evaluate the potential benefits that can be obtained when a multiprogramming mechanism was used to share the execution of multiple virtual machines on a single host. Our experiments demonstrate that having control over virtual resources and balancing the amount of CPU devoted to each virtual machine enables the development of new scheduling strategies which could be used to manage the execution of urgent jobs by adapting the system in a way that can satisfy the incoming requests and demands.

Future research includes the exploitation of previous knowledge about applications behavior, that may allow the system to perform efficient scheduling policies [13. Furthermore, we are working on defining and implementing additional feasible features of the architectural design proposed, which deal with issues such as automatic and dynamic VM activation according to the workload, the ability of virtual resources booking, or multi-core architectures exploitation in order to satisfy scientific computing intensive parallel applications.

\section{References}

1. Homepage: Spruce (November 2008), http://spruce.teragrid.org/

2. Homepage: Grid appliance user interface (November 2008), http://www.grid-appliance.org/

3. Adabala, S., Chadha, V., Chawla, P., Figueiredo, R., Fortes, J., Krsul, I., Matsunaga, A., Tsugawa, M., Zhang, J., Zhao, M., Zhu, L., Zhu, X.: From virtualized resources to virtual computing grids: the in-vigo system. Future Gener. Comput. Syst. 21, 896-909 (2005)

4. Kiyanclar, N., Koenig, G.A., Yurcik, W.: Maestro-vc: A paravirtualized execution environment for secure on-demand cluster computing. In: CCGRID 2006: Proceedings of the Sixth IEEE International Symposium on Cluster Computing and the Grid, 28 (2006)

5. Webpage: Egee - glite (November 2008), http://glite.web.cern.ch/glite/

6. Pacini, F.: Jdl attributes specification (November 2008), https://edms. cern.ch/document/590869 
7. Keahey, K., Foster, I.T., Freeman, T., Zhang, X.: Virtual workspaces: Achieving quality of service and quality of life in the grid. Scientific Programming 13, 265-275 (2005)

8. Keahey, K., Foster, I.T., Freeman, T., Zhang, X., Galron, D.: Virtual workspaces in the grid. In: Cunha, J.C., Medeiros, P.D. (eds.) Euro-Par 2005. LNCS, vol. 3648, pp. 421-431. Springer, Heidelberg (2005)

9. Homepage: Condor project (November 2008), http://www.cs.wisc.edu/condor/

10. Denham, M., Cortés, A., Margalef, T., Luque, E.: Applying a dynamic data driven genetic algorithm to improve forest fire spread prediction. In: Bubak, M., van Albada, G.D., Dongarra, J., Sloot, P.M.A. (eds.) ICCS 2008, Part III. LNCS, vol. 5103, pp. 36-45. Springer, Heidelberg (2008)

11. Homepage: Blast: Basic local alignment search tool (November 2008), http://blast.ncbi.nlm.nih.gov

12. Barham, P., Dragovic, B., Fraser, K., Hand, S., Harris, T., Ho, A., Neugebauer, R., Pratt, I., Warfield, A.: Xen and the art of virtualization. In: Proceedings of the 19th ACM Symposium on Operating Systems Principles (SOSP 2003), Bolton Landing, NY, USA. ACM, New York (2003)

13. Sherwood, T., Perelman, E., Hamerly, G., Calder, B.: Automatically characterizing large scale program behavior. In: Proceedings of the Tenth International Conference on Architectural Support for Programming Languages and Operating Systems (ASPLOS 2002), San Jose, California, pp. 45-57 (October 2002) 